\title{
Clasificación de las lagunas asociadas al Canal de Castilla (Palencia) basada en la presencia de macrófitos acuáticos y emergentes.
}

\author{
N. F. Santiago Ibarlucea ${ }^{1}$, M. Fernández-Aláez y C. Fernández-Aláez ${ }^{2}$ \\ ${ }_{1}^{1}$ Área de Botánica. E.T.S.II.AA. de Palencia. Universidad de Valladolid. Avda. de Madrid, 57. 34004 c \\ 2 Área de Ecología. Universidad de León 24071 León. degcfa@unileon.es
}

\section{RESUMEN}

Las Lagunas Marginales del Canal de Castilla constituyen unos ecosistemas de alto valor ecológico, tanto por la variada vegetación palustre y ribereña que presentan, como por las especies faunísticas que albergan, contribuyendo a aumentar la diversidad biológica y paisajística del entorno estepario donde se ubican.

A través de este estudio se ha analizado la estructura y fisonomía de las comunidades de macrófitos como base para obtener una clasificación de las lagunas. La aplicación del método de agrupación TWINSPAN establece que la permanencia y profundidad del agua son los factores diferenciadores en las lagunas marginales del Canal de Castilla y resultan determinantes de la distribución y crecimiento de la vegetación.

Palabras clave: Macrófitos Acuáticos y Emergentes, Humedales, Distribución.

\begin{abstract}
Lagoons surrounding the Canal de Castilla constitute ecosystems of high ecological value because of the variety of their marshy and riparian vegetation as well as the faunistic species present. Thus, they contribute to a greater biological diversity and broaden the range of landscapes in the steppe environments where they are found.

In this survey, the structure and composition of macrophyte communities have been analysed, as a preliminary step towards the classification of the lagoons. The application of the TWINSPAN grouping method shows that permanence and water depth are the factors that determine the distribution and development of plant communities throughout theCanal de Castilla surrounding lagoons.
\end{abstract}

Keywords: Aquatic and Emergent Macrophytes, Wetlands, Distribution.

\section{INTRODUCCIÓN}

El Canal de Castilla constituye una de las más ambiciosas e importantes obras de ingeniería hidráulica llevada a cabo entre los siglos XVIII y XIX (1753-1849), cuyo destino fue servir de vía de comunicación entre Castilla y el Cantábrico. Su trazado final tiene $207 \mathrm{~km}$ de longitud que transcurren en gran parte por la provincia de Palencia, adopta la forma de una "Y" invertida (Fig.1) y está constituido por tres ramales: Norte, Campos y Sur.

La construcción del Canal supuso grandes movimientos de tierra, ya que su profundidad oscila entre 1.8 y $3 \mathrm{~m}$ y su anchura está comprendida entre 11 y $22 \mathrm{~m}$. Esto ocasionó, en muchos casos, la formación de pronunciados terraplenes a ambos lados del Canal, que favorecieron la acumulación del agua en las depresiones del terreno junto a las márgenes del Canal y además se facilitó el estancamiento del agua por la presencia de un sustrato arcilloso impermeable.

La vegetación palustre característica de estas zonas húmedas representa un oasis de biodiversidad en las llanuras cerealistas ampliamente deforestadas de Tierra de Campos, donde se ubican. Los carrizales, juncales, espadañales, etc., confor- 


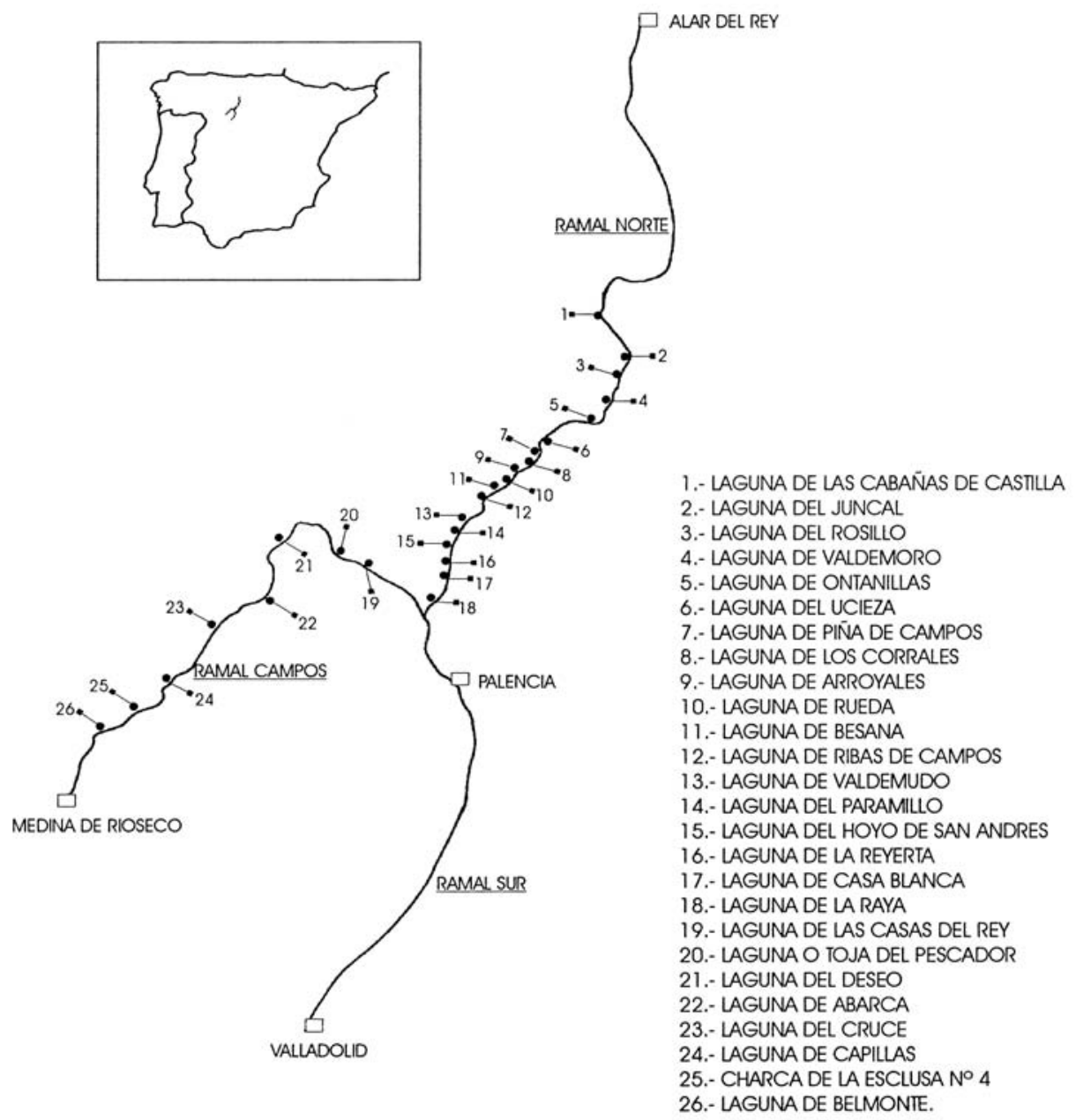

Figura 1. Área de estudio y situación de las lagunas muestreadas. Study area and location of the sampled lagoons.

man el particular aspecto de estos ecosistemas lagunares acompañados por toda una serie de plantas sumergidas, flotantes y anfibias. La importancia ecológica de estos humedales ha sido reconocida por la administración regional al haber sido incluidas todas las lagunas Marginales del Canal de Castilla en la reciente ampliación del Catálogo de Zonas Húmedas de Interés Especial en Castilla y León (Decreto 125/2001, de 19 de Abril de 2001). Muchos de estos humedales son de carácter temporal y sufren fuertes fluctuaciones estacionales, llegando incluso a secarse en el periodo estival. Esta circunstancia condiciona la composición de las comunidades vegetales palustres que los colonizan, experimentando diversos grados de adaptación al medio (Cirujano, 2000; Cirujano et al., 2000). Varios autores han puesto de manifiesto que algunas especies de humedales son especialmente sensibles a cambios en el régimen de agua, la altura que presente el nivel del agua y la duración de la inundación, lo que determinará la localización exacta de las especies en la comunidad del humedal (Margalef Mir, 1981; Denny, 1992; Fernández Aláez et al., 1999).

El objetivo de este estudio ha sido analizar la estructura y fisonomía de las comunidades de macrófitos en las lagunas asociadas al Canal de Castilla, tratando de obtener una clasificación de los humedales estudiados en relación con la vegetación macrofítica acuática y emergente. 


\section{ÁREA DE ESTUDIO}

El Canal de Castilla queda enmarcado en la cuenca terciaria del Duero, dentro de la región geográfica denominada "Tierra de Campos", en el centro de la meseta septentrional, que se extiende por el sector central y suroccidental de Palencia, limitando al norte con los páramos detríticos y al sur con los páramos calcáreos. Las lagunas objeto de estudio están distribuidas a lo largo de los ramales Norte y Campos del Canal y todas ellas se sitúan dentro de la provincia de Palencia.

La red hidrográfica que tiene relación con el Canal de Castilla está formada por dos tipos de ríos: unos más caudalosos, como el río Carrión y el río Pisuerga, de los cuales el Canal aprovecha su caudal; y otros cortos y de caudal exiguo, ya que no reciben aportes considerables, como son el Valdeginate, Retortillo, Cueza, Ucieza y Vallarna.

La climatología del área de estudio pone de manifiesto una acusada continentalidad, propia del clima mediterráneo (inviernos largos y fríos que alternan con veranos cortos y cálidos), caracterizada por la escasez e irregularidad interanual de las precipitaciones, en torno a los $400 \mathrm{~mm}$ anuales, que aumentan de sur a norte. Se aprecia un doble máximo de precipitaciones, en primavera y otoño, y un mínimo estival en los meses de Julio y Agosto.

De las características morfométricas de este conjunto lagunar (Tabla 1), podemos destacar que la mayor parte de las formaciones palustres

Tabla 1. Características morfométricas de las Lagunas Marginales del Canal de Castilla. Morphometric characteristics of the marginal lagoons of Canal de Castilla.

\begin{tabular}{|c|c|c|c|c|c|c|}
\hline LAGUNAS & $\begin{array}{l}\text { SUPERFICIE } \\
\text { (ha) }\end{array}$ & $\begin{array}{l}\text { PERIMETRO } \\
\text { (m) }\end{array}$ & $\begin{array}{l}\text { LONGITUD } \\
\text { MAXIMA (m) }\end{array}$ & $\begin{array}{c}\text { ANCHURA } \\
\text { MAXIMA (m) }\end{array}$ & $\begin{array}{r}\text { PROFUNDIDAD } \\
\text { MAXIMA (cm) }\end{array}$ & $\begin{array}{c}\text { PERSISTENCIA } \\
(*)\end{array}$ \\
\hline LAS CABAÑAS & 1.35 & 516.15 & 172 & 195 & 200 & Semipermanente (4) \\
\hline EL JUNCAL & 0.76 & 367.32 & 113 & 106 & 120 & Temporal (3) \\
\hline EL ROSILLO & 0.47 & 317.11 & 111 & 84 & 80 & Temporal (3) \\
\hline VALDEMORO & 8.34 & 2021.60 & 633 & 375 & 100 & Temporal (3) \\
\hline ONTANILLAS & 1.35 & 627.25 & 223 & 106 & 250 & Permanente (5) \\
\hline UCIEZA & 1.14 & 1049.50 & 489 & 28 & 200 & Permanente (5) \\
\hline PIÑA DE CAMPOS & 1.25 & 654.10 & 73 & 260 & 30 & Temporal (3) \\
\hline LOS CORRALES & 2.00 & 1298.28 & 717 & 76 & 60 & Temporal (3) \\
\hline ARROYALES & 0.56 & 321.20 & 105 & 77 & 30 & Temporal (3) \\
\hline RUEDA & 3.81 & 1191.47 & 456 & 106 & 200 & Semipermanente (4) \\
\hline BESANA & 4.70 & 990.12 & 334 & 192 & 150 & Temporal (3) \\
\hline RIBAS DE CAMPOS & 8.20 & 2108.50 & 382 & 466 & 290 & Permanente (5) \\
\hline VALDEMUDO & 29.35 & 3454.08 & 1.500 & 259 & 250 & Permanente (5) \\
\hline PARAMILLO & 1.65 & 1097.46 & 364 & 67 & 70 & Permanente (5) \\
\hline HOYO DE SAN ANDRES & 1.04 & 454.49 & 87 & 136 & 200 & Permanente (5) \\
\hline LA REYERTA & 1.20 & 477.92 & 143 & 137 & 70 & Semipermanente (4) \\
\hline CASA BLANCA & 1.45 & 694.79 & 228 & 89 & 70 & Semipermanente (4) \\
\hline LA RAYA & 3.29 & 1111.31 & 447 & 85 & 70 & Semipermanente (4) \\
\hline CASAS DEL REY & 0.52 & 280.17 & 58 & 91 & 200 & Semipermanente (4) \\
\hline TOJA DEL PESCADOR & 0.96 & 413.00 & 90 & 119 & 30 & Temporal (3) \\
\hline EL DESEO & 2.65 & 1102.05 & 396 & 162 & 120 & Semipermanente (4) \\
\hline ABARCA & 2.05 & 1043.54 & 448 & 63 & 60 & Temporal (3) \\
\hline EL CRUCE & 1.95 & 1267.66 & 546 & 73 & 150 & Permanente (5) \\
\hline CAPILLAS & 0.74 & 579.20 & 263 & 34 & 50 & Temporal (3) \\
\hline ESCLUSA No 4 & 0.33 & 289.43 & 119 & 33 & 50 & Muy Temporal (2) \\
\hline BELMONTE & 2.22 & 1392.67 & 587 & 59 & 150 & Permanente (5) \\
\hline
\end{tabular}

(*) Entre paréntesis se indica el valor numérico asignado a cada laguna según la persistencia del agua. 
estudiadas tienen una superficie entre 1 y 3 ha, aunque el rango es muy amplio ya que 7 lagunas no superan $1 \mathrm{ha}$, mientras que existen 6 con una superficie mayor de 3 ha. Por lo que se refiere a la persistencia del agua en la cubeta lagunar, 8 lagunas se pueden considerar permanentes e igualan o superan $1.5 \mathrm{~m}$ de profundidad máxima; otro grupo de lagunas se han clasificado como semipermanentes, entendiendo con ello que el agua permanece en la cubeta casi todo el año. Con un periodo de inundación de 6 a 9 meses y correspondiendo a la categoría de lagunas temporales, figuran un total de 12 lagunas, cuya profundidad máxima es inferior a $1.5 \mathrm{~m}$, y finalmente otra permanece inundada entre 2 y 6 meses, y se ha clasificado como muy temporal. Generalmente se comprueba que las lagunas con una superficie inferior a 1 ha son temporales o muy temporales.

\section{METODOLOGÍA}

A partir de la información obtenida de distintas fuentes (mapas del Canal de Castilla a escala 1/10.000 elaborados por la Confederación Hidrográfica del Duero en el año 1992; fotografía aérea del I.N.G., vuelo nacional y escala 1/30.000, de Agosto de 1984; ortofotografía a escala $1 / 5.000$, facilitada por la Dirección General del Centro de Gestión Catastral de Madrid; mapas a Escala 1:50.000 del Servicio Geográfico del Ejército; comunicaciones personales) se seleccionaron 26 humedales, que cumplían los criterios básicos establecidos para su selección: poseer una cubeta bien definida que los separase claramente de los sistemas terrestres adyacentes, una vegetación típicamente palustre y una profundidad suficiente para permitir el almacenamiento del agua durante, al menos, dos meses al año.

El muestreo de la vegetación macrófita en las lagunas seleccionadas se realizó entre los meses de Junio y Septiembre a lo largo de 4 años (1995 - 1999). Durante este periodo de tiempo se realizó un muestreo de la vegetación palustre mediante la realización de un número variable de transectos en función de la extensión y características particulares de cada laguna que se completó con un muestreo semicuantitativo, donde se estimó de forma visual el porcentaje de cobertura de las especies presentes mediante la siguiente escala de valoración: $1=$ Presencia, $2=1-3 \%, 3=3-6 \%, 4=6-12 \%, 5=12-25 \%$, $6=25-50 \%$ y $7=50-100 \%$.

A partir de los datos obtenidos en el muestreo se elaboró una matriz de datos en la que se incluyeron 70 especies (35 hidrófitos y 35 helófitos) presentes en las 26 lagunas seleccionadas en el estudio (Tabla 2). Sobre esta matriz se aplicó un análisis de clasificación TWINSPAN (Hill, 1980), excluyendo previamente las especies de helófitos con valor 1 y presentes en una sola laguna. Con el fin de determinar la posible influencia del nivel de permanencia del agua en la laguna sobre la estructura de la comunidad de macrófitos se aplicó el test no paramétrico de Spearman.

\section{RESULTADOS}

En la figura 2 se muestra la clasificación de las lagunas asociadas al Canal de Castilla obtenida al aplicar el análisis TWINSPAN. Esta clasificación responde a tres propiedades de los ecosistemas palustres: la persistencia del agua en la cubeta, la profundidad del agua, y el tipo de crecimiento y desarrollo de cada especie. La primera división clasifica a las lagunas en función de la persistencia del agua y separa un grupo de humedales cuyas especies indicadoras son Alisma plantago-aquatica, Rumex conglomeratus y Baldellia ranunculoides. Estos taxones corresponden a lagunas con un régimen de inundación temporal o muy temporal, aunque también aparecen en algunas lagunas semipermanentes pero que llegan a secarse durante el verano en los años de pertinaz sequía (laguna de Casas del Rey, laguna de las Cabañas de Castilla, laguna de Rueda y laguna del Deseo). Aparecen también ligados a este grupo algunos hidrófitos como Hippuris vulgaris $\mathrm{y}$ Polygonum amphibium. El segundo grupo de 
Tabla 2. Listado de plantas (hidrófitos y helófitos) presentes en las lagunas asociadas al Canal de Castilla sobre los que se aplicó el análisis TWINSPAN. List of plants (hydrophytes and helophytes) present in the lagoons asociated with the Canal de Castilla, over which the grouping method TWINSPAN was applied.

\section{HIDRÓFITOS}

Chara aspera Deth. Ex Willd

Chara fragilis Desv. (Chara globularis Thuill. var. globularis)

Chara hispida L.

Chara hispida L. var. major (Hartm.) R.D. Wood

Chara vulgaris L. var. contraria (A. Braun ex Kütz) J.A. Moore

Chara vulgaris L. var. longibracteata (Kütz) J. Grov. \& Bull. Web.

Chara vulgaris L. var. vulgaris

Nitella mucronata (A. Braun) Miquel

Nitella opaca (Bruz.) Agardh

Nitella translucens (Pers.) Agardh

Tolypella glomerata (Desv.) Leonh.

Amblystegium humile (P. Beauv.) Crundw. (*)

Amblystegium serpens (Hedw.) B., S. (*)

Brachythecium rutabulum (Hedw.) B., S. \& G (*)

Bryum pseudotriquetrum (Hedw.) Gaertn., Meyer \& Scherb. (*)

Drepanocladus aduncus (Hedw.) Warnst. (*)

Homalothecium lutescens (Hedw.) Robins (*)

Ceratophyllum demersum $\mathrm{L}$.

Groenlandia densa (L.) Four.

Hippuris vulgaris L.

Lemna minor L.

Myriophyllum spicatum $\mathrm{L}$.

Myriophyllum verticillatum L.

Polygonum amphibium L.

Potamogeton crispus L.

Potamogeton gramineus L.

Potamogeton lucens L.

Potamogeton natans L.

Potamogeton pectinatus L.

Ranunculus aquatilis L.

Ranunculus peltatus Schrank

Ranunculus trichophyllus Chaix

Utricularia australis R. Br.

Zannichellia palustris L.

Zannichellia peltata Bertol.

\section{HELÓFITOS}

Alisma lanceolatum With.

Alisma plantago-aquatica L.

Apium nodiflorum (L.) Lag.

Baldellia ranunculoides (L.) Parl.

Carex acutiformis Ehrh.

Carex flacca Schreber

Carex pseudocyperus L.

Carex riparia Curtis

Damasonium polyspermum Cosson

Eleocharis palustris (L.) Roemer \& Schultes

Eleocharis uniglumis (Link) Schultes

Galium palustre L.

Glyceria fluitans (L.) R. Br.

Iris pseudacorus L.

Juncus acutus L.

Juncus articulatus L.

Juncus bulbosus L.

Juncus maritimus Lam.

Lycopus europaeus L.

Lysimachia vulgaris L.

Lythrum salicaria L.

Mentha aquatica Salzm. Ex Sprengel

Mentha pulegium L.

Nasturtium officinale R. Br. In Aiton

Phalaris arundinacea $\mathrm{L}$.

Phragmites australis (Cav.) Trim. Ex Stendel

Rumex conglomeratus Murray

Rumex palustris $\mathrm{Sm}$.

Scirpus lacustris L. subsp. lacustris

Scirpus maritimus L.

Sparganium erectum L. subsp. neglectum (B.) Sch. \& Thell.

Typha domingensis (Pers.) Stendel

Typha latifolia L.

Veronica anagallis-aquatica $\mathrm{L}$.

Veronica scutellata L.

(*) Las especies de musgos se han incluido en el grupo de hidrófitos.

lagunas dentro de la primera división tiene como especies indicadoras: Lysimachia vulgaris, Sparganium erectum, Carex acutiformis, Carex riparia y Carex pseudocyperus. Estas especies caracterizan la zona ecotónica de un grupo de lagunas permanentes (con profundidad máxima de 3 metros) o que habitualmente están inundadas, aunque descienda considerablemente el nivel del agua en la época estival, concentrándose en pequeñas charcas interiores o en arroyos que circundan el humedal.

En la segunda división aparecen dos grupos de lagunas en función de la capacidad de adaptación de los grandes helófitos a las fluctuacio- 


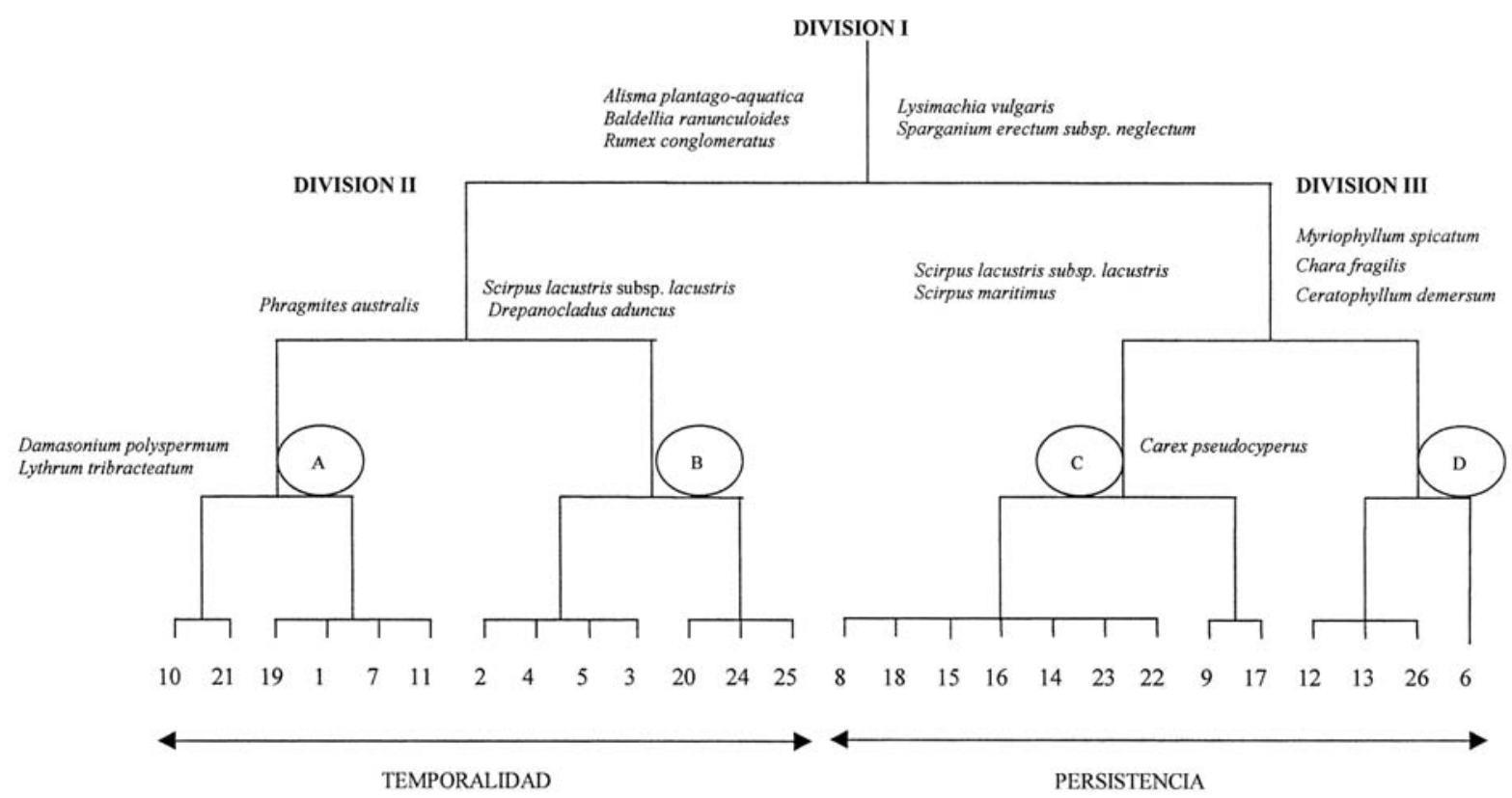

Figura 2. Clasificación TWINSPAN de las Lagunas Marginales del Canal de Castilla basada en el porcentaje de cobertura de las especies de macrófitos incluyendo las especies indicadoras. Se indican los números de las lagunas, cuya denominación aparece en la figura 1. TWINSPAN classification of the Marginal Lagoons of the Canal de Castilla based on cover percentage of the macrophyte species including indicator species. The numbering of the lagoons is shown, following the codes established in figure. 1

nes hídricas de la cubeta lagunar (Fig. 2). Así, se generó un primer grupo de lagunas (Grupo A: 10, 21, 19, 1, 7, 11) con Phragmites australis como especie indicadora y en las que la profundidad media máxima del agua fue algo mayor $(155 \mathrm{~cm})$. Un segundo grupo (Grupo B: $2,4,5,3,20,24,25)$ correspondió a lagunas con menor profundidad media máxima $(97 \mathrm{~cm})$, siendo la especie más representativa Scirpus lacustris subsp. lacustris.

La tercera división está relacionada con el nivel del agua en las lagunas de carácter permanente. Así, aparece un grupo formado por cuatro lagunas (Grupo D: 12, 13, 26, 6), que presentan mayor profundidad media máxima $(222 \mathrm{~cm})$, ya sea en toda su superficie o en reducidas charcas interiores, y cuya especie indicadora fue Myriophyllum spicatum, acompañada por Ceratophyllum demersum y Chara fragilis. El otro grupo de lagunas (Grupo C: 8, $18,15,16,14,23,22,9,17)$, con un nivel de agua más fluctuante y con menor profundidad media máxima $(87 \mathrm{~cm})$, estuvo ampliamente colonizado por Scirpus lacustris subsp. lacustris y Scirpus maritimus.

En la Tabla 3 se indican los valores medios de distintas variables morfométricas (grado de persistencia, superficie, profundidad máxima, clorofila a) y de riqueza (hidrófitos, helófitos) correspondientes a los cuatro grupos principales de lagunas que se derivan del TWINSPAN. En las lagunas incluidas en el grupo $\mathrm{D}$, que son las de mayor superficie y profundidad, y en las que el agua persiste durante más tiempo en la cubeta, la riqueza de hidrófitos y helófitos fue claramente superior al resto de las lagunas. Esta característica ha determinado la existencia de una correlación significativa del grado de persistencia con el número de especies de hidrófitos $\left(\mathrm{r}_{\mathrm{S}}=0.56, \mathrm{p}<0.05\right)$ y de helófitos $\left(\mathrm{r}_{\mathrm{S}}=0.42\right.$, $\mathrm{p}<0.05$ ), ya que para los grupos $\mathrm{A}, \mathrm{B}$ y $\mathrm{C}$ del TWINSPAN las diferencias en el nivel de permanencia del agua no parecen haber sido determinantes del valor alcanzado por la riqueza de ambos tipos de macrófitos. Si se considera la riqueza de especies por unidad de superficie, no 
Tabla 3. Valores medios y rango de las variables morfométricas y de riqueza correspondientes a los cuatro grupos de lagunas obtenidos en el TWINSPAN. Average values and range of the morphometric variables and richness for the four groups obtained with TWINSPAN.

\begin{tabular}{|c|c|c|c|c|c|c|c|}
\hline GRUPOS (*) & $\begin{array}{c}\mathrm{N}^{0} \mathrm{DE} \\
\text { LAGUNAS }\end{array}$ & $\begin{array}{c}\text { GRADO DE } \\
\text { PERSISTENCIA }\end{array}$ & $\begin{array}{l}\text { SUPERFICIE } \\
\text { (ha) }\end{array}$ & $\begin{array}{l}\text { PROFUNDIDAD } \\
\text { MÁXIMA (cm) }\end{array}$ & $\begin{array}{l}\text { CLOROFILA } a \\
(\mathrm{mg} / \mathrm{l})(* *)\end{array}$ & $\begin{array}{c}\text { RIQUEZA } \\
\text { HIDRÓFITOS }\end{array}$ & $\begin{array}{l}\text { RIQUEZA } \\
\text { HELÓFITOS }\end{array}$ \\
\hline A & 6 & $\begin{array}{c}3 \\
(3-4)\end{array}$ & $\begin{array}{c}2.38 \\
(0.52-4.7)\end{array}$ & $\begin{array}{c}155 \\
(30-200)\end{array}$ & $\begin{array}{c}27.74 \\
(2-56.61)\end{array}$ & $\begin{array}{c}7 \\
(3-11)\end{array}$ & $\begin{array}{c}13 \\
(6-20)\end{array}$ \\
\hline B & 7 & $\begin{array}{c}3 \\
(2-3)\end{array}$ & $\begin{array}{c}1.85 \\
(0.33-8.34)\end{array}$ & $\begin{array}{c}97 \\
(30-200)\end{array}$ & $\begin{array}{c}6.09 \\
(1.79-19.58)\end{array}$ & $\begin{array}{c}5 \\
(2-10)\end{array}$ & $\begin{array}{c}12 \\
(10-15)\end{array}$ \\
\hline $\mathrm{C}$ & 9 & $\begin{array}{c}4 \\
(3-5)\end{array}$ & $\begin{array}{c}1.69 \\
(0.56-3.29)\end{array}$ & $\begin{array}{c}87 \\
(30-200)\end{array}$ & $\begin{array}{c}5.42 \\
(0.57-12.2)\end{array}$ & $\begin{array}{c}5 \\
(1-8)\end{array}$ & $\begin{array}{c}13 \\
(10-16)\end{array}$ \\
\hline D & 4 & $\begin{array}{c}5 \\
(5-5)\end{array}$ & $\begin{array}{c}10.23 \\
(1.14-29.35)\end{array}$ & $\begin{array}{c}222 \\
(150-290)\end{array}$ & $\begin{array}{c}5.52 \\
(2.97-8.06)\end{array}$ & $\begin{array}{c}11 \\
(8-16)\end{array}$ & $\begin{array}{c}17 \\
(11-30)\end{array}$ \\
\hline
\end{tabular}

(*) Grupo A: lagunas n $n^{\circ}$ 10, 21, 19, 1, 7, 11.

(**) Valores medios del año 1996

Grupo B: lagunas $\mathrm{n}^{\circ}$ 2, 4, 5, 3, 20, 24, 25.

Grupo C: lagunas no $8,18,15,16,14,23,22,9,17$.

Grupo D: lagunas $n^{\circ} 12,13,26,6$.

se obtienen valores superiores en las lagunas permanentes y de mayor tamaño que en las estacionales y de menor tamaño. Por otro lado, no se observa una relación clara entre los valores de clorofila $a$ (indicadores del grado de eutrofia de un humedal) y la presencia de hidrófitos, ya que las lagunas más eutróficas (grupo A) tienen valores de riqueza en hidrófitos similares a las menos eutróficas (grupos B y C).

\section{DISCUSIÓN}

Las lagunas Marginales del Canal de Castilla forman un conjunto de ecosistemas palustres en los que la alimentación hídrica procede tanto de escorrentías superficiales como de las infiltraciones del propio Canal. Existe una gran variedad de lagunas en cuanto a la persistencia del agua en sus cubetas (lagunas permanentes, semipermanentes, temporales y muy temporales) y una diversificada vegetación de macrófitos que se disponen siguiendo un gradiente de inundación.

Coincidiendo con lo señalado por Fernández Aláez (1984) y Fernández Aláez et al. (1984, 1999) para lagunas del sureste de la provincia de León, la profundidad del agua y su variabili- dad estacional son los factores más importantes que afectan a la distribución y crecimiento de la vegetación macrófita en las lagunas Marginales del Canal de Castilla y condicionan, en cierta medida, la riqueza de especies de helófitos e hidrófitos. Los resultados obtenidos ponen de manifiesto también lo indicado por Denny (1992), en relación a que algunas especies de humedales son especialmente sensibles a cambios en el régimen de agua. La altura que presente el nivel del agua y la duración de la inundación determinarán la localización de cada una de las especies en la comunidad del humedal. En la mayoría de las lagunas temporales o muy temporales asociadas al Canal, que al menos permanecieron secas durante la estación estival, la zona ecotónica estuvo marcada por helófitos como Alisma plantago-aquatica y Baldellia ranunculoides, que se desarrollaron en la época de llenado, generalmente en primavera. El régimen de inundación temporal o muy temporal de estas lagunas determina la presencia de las especies citadas, con un periodo vegetativo corto (anuales o bianuales) y un sistema radicular no rizomatoso; si bien pueden aparecer hidrófitos que presentan un largo rizoma rastrero y pueden crecer como helófitos en ausencia 
de lámina de agua, llegando a formar verdaderos tapices en suelos desecados pero que mantienen la humedad por la presencia de abundante musgo (Drepanocladus aduncus). Sin embargo, en las lagunas permanentes o semipermanentes, que mantienen agua a lo largo de su ciclo anual, aunque puedan sufrir fluctuaciones importantes dependiendo de su profundidad, la zona de transición entre el medio acuático y terrestre estuvo colonizada por helófitos perennes, como Sparganium erectum subsp. neglectum, Lysimachia vulgaris y diversas especies de Carex, dotados de una compleja estructura rizomatosa que los ancla fuertemente al sustrato, permitiéndoles una buena adaptación al ambiente acuático y terrestre.

Dado que una gran parte del conjunto lagunar estudiado presentó aguas de poca profundidad, los grandes helófitos del tipo de Scirpus lacustris subsp. lacustris y Phragmites australis, cubrieron prácticamente todo el interior lagunar gracias a los potentes rizomas que penetran en el sedimento, y les otorgan su fisonomía peculiar, contribuyendo, además, con su importante biomasa vegetal a colmatar la cubeta lagunar. Tal como afirma Margalef Mir (1981), se trata de plantas de gran poder invasor, dado que sus grandes rizomas y a veces largos estolones aéreos les permiten competir favorablemente con otras especies. En el caso de Phragmites la masa foliar aérea ayuda a crear las condiciones de sombreado precisas para evitar el establecimiento de otras especies bajo sus tallos. Sin embargo, estos dos grandes helófitos no se reparten por igual en todas las lagunas estudiadas. Phragmites australis estuvo ausente en lagunas temporales con pronunciada sequía estival, en las que creció abundante musgo (Drepanocladus aduncus), que llegó a formar un alfombrado de considerable espesor $(10-15 \mathrm{~cm})$. La masa musgosa parece fundamental para mantener la humedad de la capa freática en la superficie lagunar. Entre sus hojitas se desarrollaron bien los altos tallos de Scirpus lacustris y al mismo tiempo hicieron posible el mantenimiento de algunos hidrófitos (Hippuris vulgaris y Polygonum amphibium) como plantas anfibias. Por tanto, se deduce que
Phragmites australis se desarrolla mejor en lagunas que presentan mayor nivel y permanencia del agua en sus cubetas, mientras que Scirpus lacustris quedaría competitivamente desplazado por Phragmites australis; manteniendo una escasa y dispersa presencia. Esta observación es confirmada por Weisner $(1987,1991)$, aunque no coincide con otras investigaciones (Ekstam \& Weisner, 1991; Weisner et al., 1993), que apuntan a que en aguas permanentes Phragmites australis presenta menor poder colonizador al tener que confiar en la expansión vegetativa, por sus rizomas, que sólo puede ocurrir en una proporción limitada, mientras que Scirpus lacustris puede extenderse más rápidamente por el establecimiento de semillas.

Se puso también de manifiesto a través de nuestros resultados la influencia de la profundidad del agua en las lagunas permanentes o semipermanentes (generalmente inundadas pero con riesgo de desecación) sobre la distribución de la vegetación acuática. En lagunas permanentes, como Ribas, Valdemudo, Belmonte y Ucieza, donde el agua mantiene un nivel estacional constante de hasta 3 metros, es posible la instalación de una comunidad de hidrófitos sumergidos en la que Myriophyllum spicatum alcanza un buen desarrollo. La importante biomasa desarrollada por este hidrófito, que domina ampliamente la lámina de agua desprovista de vegetación emergente, impide el asentamiento de otros macrófitos sumergidos, en especial de carófitos. El hecho de haber encontrado de forma ocasional algún ejemplar aislado de Chara fragilis cerca de la orilla, donde la profundidad es menor, puede indicar una anterior presencia más significativa de este carófito en los humedales, ya que como sostienen Crawford (1977) y Blindow (1992a), los carófitos están entre los primeros macrófitos sumergidos que colonizan los pequeños cuerpos de agua, pero a menudo son desplazados por las angiospermas. Un aumento en la turbidez y la cantidad de nutrientes son probablemente los factores que han podido influir de forma notoria en la desaparición de los carófitos, como demuestran múltiples estudios realizados en ecosistemas palustres de todo 
el mundo. Así, varios autores (Madsen et al., 1989; Madsen et al 1991; Blindow, 1992b), sostienen que la forma de crecimiento de Myriophyllum spicatum, con el alargamiento característico de sus brotes, permite crear un denso dosel superior que contribuye sustancialmente a reducir la intensidad de luz disponible para las plantas bajo el dosel. Además la densa masa radicular formada por Myriophyllum spicatum, puede causar la exclusión de algunas especies. $\mathrm{Si}$ a esto añadimos la prolífica formación de fragmentos del tallo, como método eficiente de reproducción y dispersión (Smith, 1963) y una habilidad para explotar hábitat perturbados, podemos tener los elementos clave para explicar su éxito colonizador en los humedales.

\section{CONCLUSIONES}

Se concluye, pues, que la permanencia del agua y la profundidad que alcanza en la cubeta son los factores determinantes de la distribución y riqueza de especies dentro del humedal y además afectan a la dinámica de la vegetación acuática otorgándole ciertas particularidades relacionadas con la sustitución de unas especies por otras.

De los resultados obtenidos al tratar de establecer las correlaciones entre diferentes parámetros morfométricos (grado de persistencia, superficie, profundidad máxima, clorofila $a$ ) y de riqueza de especies (hidrófitos, helófitos) que justifiquen la aparición de los cuatro grandes grupos de lagunas formados en el TWINSPAN, se deduce que no existe una agrupación clara de los humedales estudiados en función de los parámetros considerados a excepción de la profundidad máxima y la riqueza de hidrófitos, $y$, como era de esperar, las lagunas más profundas presentaron un buen desarrollo de la flora acuática sumergida.

\section{BIBLIOGRAFÍA}

BLINDOW, I. 1992a. Decline of charophytes during eutrophication: comparison with angiosperms. Freshwat. Biol., 28: 9-14.
BLINDOW, I. 1992b. Long - and short - term dynamics of submerged macrophytes in two shallow eutrophic lakes. Freshwat. Biol., 28: 15-27.

CRAWFORD, S. A. 1977. Chemical, physical and biological changes associated with Chara succession. Hidrobiologia, 55: 209-218.

CIRUJANO, S. 2000. Flora acuática de las lagunas y zonas húmedas españolas. Quercus, 171: 38-44.

CIRUJANO, S. \& N. F. SANTIAGO IBARLUCEA. 2000. Caracterización botánica de la laguna de Valdemudo (Becerril de Campos, Palencia). Anales Jard. Bot. Madrid, 57: 441-444.

DENNY, P. 1992. Water management strategies for the conservation of wetlands. Anual Symposium on the Management of Scarce Water Resources. Brighton.

EKSTAM, B. \& S. E. B. WEISNER. 1991. Dynamics of emergent vegetation in relation to open water of shallow lakes. In: Wetland Management and Restoration. Proc. Workshop, Sweden 1990. C.M. Finlayson and T Larsson (eds.), 46-54. Swedish Environmental Proctection Agency, Solna, Sweden.

FERNÁNDEZ ALÁEZ, C., M. FERNÁNDEZ ALÁEZ \& E. BÉCARES. 1999. Influence of water level fluctuation on the structure and composition of the macrophyte vegetation in two small temporary lakes in the northwest of Spain. In: Biology, Ecology and Management of Aquatic Plants. J.M. Caffrey, P.R.F. Barret, M.T. Ferreira, I.S. Moreira, K.J. Murphy \& P.M. Wade (eds). Kluver Academic Publishers. Netherlands. Hydrobiologia, 415: 155-162.

FERNÁNDEZ ALÁEZ, M. 1984. Distribución de la vegetación macrófita y evaluación de factores ecológicos en sistemas leníticos de la provincia de León. Tesis doctoral. Universidad de León. 307 pp.

FERNÁNDEZ ALÁEZ, M., E. LUIS \& C. FERNÁNDEZ ALÁEZ. 1984. Distribución de la vegetación macrofítica en las Lagunas de Chozas de Arriba, León. Limnetica, 1: 101-110.

HILL, M. O. \& H. G. GAUCH. 1980. Detrended correspondence analysis, an improved ordination technique. Vegetatio, 42: 47-58.

MADSEN, J. D., C.F. HARTLED \& C. W. BOYLEN. 1989. A photosynthetic survey of seven submersed aquatic macrophyte species from Lake George, New York..Rensselaer Fresh Water Institute Report. 21 pp.

MADSEN, J. D., J. W. SUTHERLAND, J. A. BLOOMFIELD, J. A. ROY, L. W. EICHLER \& C. W. BOYLEN. 1991. The decline of native vegetation 
under dense Eurasian water milfoil canopies. $J$. Aquat. Plant Mgmt., 29: 94-99.

MARGALEF MIR, R. 1981. Distribución de los macrófitos de las lagunas dulces y salobres del Este y Noreste de España y dependencia de la composición química del medio. Serie Universitaria 157. Fundación Juan March. Madrid: 21-30

SMITH, G. E. 1963. Control of Eurasian water milfoil (Myriophyllum spicatum) in TVA reservoirs. Proc. Southeastern Weed Control Conf., 16: 351-353.
WEISNER, S. E. B. 1987. The relation between wave exposure and distribution of emergent vegetation in a eutrophic lake. Freshwat. Biol., 18: 537-544.

WEISNER, S. E. B. 1991. Within-lake patterns in depth penetration of emergent vegetation. Freshwat. Biol., 26: 133-142.

WEISNER, S. E. B., W. GRANELI \& B. EKSTAM. 1993. Influence of submergence on growth of seedling of Scirpus lacustris and Phragmites australis. Freshwat. Biol., 29: 371-375. 